\title{
Breast Carcinoma Grade G2
}

National Cancer Institute

\section{Source}

National Cancer Institute. Breast Carcinoma Grade G2. NCI Thesaurus. Code C147917.

An intermediate combined histologic grade (moderately favorable), SBR score of 6-7 points. 